[13] G. E. Bloom, A. Eris, and R. Ruble, "Modelling, analysis, and design of a multi-output Cuk converter," in Proc. Seventh $\mathrm{Na}$ tional Solid-State Power Conversion Conf., pp. I1.1-I1.19, Mar. 1980.

[14] G. E. Bloom and A. Eris, "Modelling and analysis of a multioutput Cuk converter," presented at IEEE Power Electronics Specialists Conf., June 1980, A tlanta, GA.

[15] W. T. McLyman, Transformer and Inductor Design Handbook. New York: Marcel Dehker.

[16] S. Cuk and R. D. Middlebrook, "Dc-to-dc switching converter," U.S. Patent 4,184,197, January 15, 1980 . Foreign patents pending.

[17] S. Cuk, "Push-pull switching power amplifier," U.S. Patent $4,186,437$, Jan. 29, 1980. Foreign patents pending.

[18] - "Dc-to-dc switching converter with zero input and output current ripple and integrated magnetics circuits," U.S. Patent Application, March 30, 1979.

[19] S. Cuk and R. D. Middlebrook, "Dc-to-dc converter having re- duced ripple without need for adjustments," U.S. Patent Applicacation, June 15, 1979.

[20] P. Wood, "General theory of switching power converters," in IEEE Power Electronics Specialists Conf., 1979 Record, pp. 3-10 (IEEE Publication 79CH1461-3 AES).

[21] S. Cuk, "Switching dc-to-dc converter with zero input or output current ripple," in IEEE Ind. Appl. Soc. Annu. Meeting, 1978 Record, pp. 1131-1145, (IEEE Publication 78CH1346-61A).

[22] H. Hirayama, "Simplifying switched mode converter design with a new variable leakage transformer topology," in Proc. Seventh National Solid-State Power Conversion Conf. (Powercon 7), pp. E1.1-E1.10, Mar. 1980.

[23] R. P. Massey and E. C. Snyder, "High-yoltage single-ended dc-dc converter," in IEEE Power Electronics Specialists Conf., 1977 Record, pp. 156-159.

[24] R. D. Middlebrook, "Modelling and Design of the Cuk Converter," in Proc. Sixth National Solid-State Power Conversion Conf. (Powercon 6), pp. G3.1-G3.14, May 1979.

\title{
New Magnetic Structures for Switching Converters
}

\author{
SLOBODAN ĆUK, MEMBER, IEEE
}

\begin{abstract}
In the past the majority of power processing applications have been centered around a very few standard switching converter topologies. Recently, a number of new converter topologies have been proposed in order to find the best possible electrical interconnection of power processing elements: switches, storage components, and transformers, that would yield the highest efficiency and best performance. However the equally important and complementary problem of their best magnetic interconnection has been completely overlooked. In some new converter structures, the nature of the switching process and existing waveforms allows integration of previously separate inductors and transformers into a single magnetic structure. Several such magnetic core structures are proposed and analyzed, which lead to further converter simplifications and performance improvements.
\end{abstract}

\section{INTRODUCTION}

$\mathrm{U}$ $P$ UNTIL two decades ago, only a small number of standard switching configurations have been in widespread use, such as the basic buck, boost, buck-boost, flyback, buck-forward, push-pull, half-bridge, and full-bridge switching converters. However each of these configurations had both theoretical and practical disadvantages, often requiring addition of some extra components (such as input filtering in the buck or buck-boost derived family of converters, flux sensing to prevent saturation in push-pull converters, etc.) to cure

Manuscript received August 13, 1982; revised November 23, 1982. This paper was presented at the Power Conversion International Conference, Munich, September 14-17, 1981. This work was supported by the Office of Naval Research, Washington, DC, under Contract N00014-78-C-0757.

The author is with the California Institute of Technology, 116-81 Caltech, Pasadena, CA 91125. some of their deficiencies. In fact, some of the early "switcher" designs have been so notorious in noise generation that the whole field of switching power supplies received a bad name initially.

Early attempts to cure some of these noise performance problems by adding brute-force filtering and heavy shielding resulted in designs almost as heavy and bulky as their linear power supplies predecessors. Fortunately, space applications and their requirements for small size, weight, and highest efficiency maintained a strong interest and sponsored innovations in switching power supplies. Just within the last decade a number of new switching configurations mushroomed, primarily because of the need to come up with more efficient and "quiet" converter topologies.

The prior effort in devising new, better converter topologies has been documented in several Ph.D. dissertations [1], [2], in a two-volume book Advances in Switched-Mode Power Conversion [3], [4], and in a series of patents [5]-[8]. Throughout this research effort the emphasis was on the topology of the electrical interconnections among various components (switches, capacitors, inductors, and transformers), as illustrated on the general block diagram of Fig. 1(a). Several new converter configurations [1], [4] have been proposed and thoroughly analyzed [1], [3] .

Recognition of some of the unique features of the characteristic switching waveforms in these converters led to the establishment of two general concepts: the coupled-inductor technique and the integrated magnetics method. Although general in nature, they are illustrated in Section II on the Cuk converter where they originated. These techniques opened up 


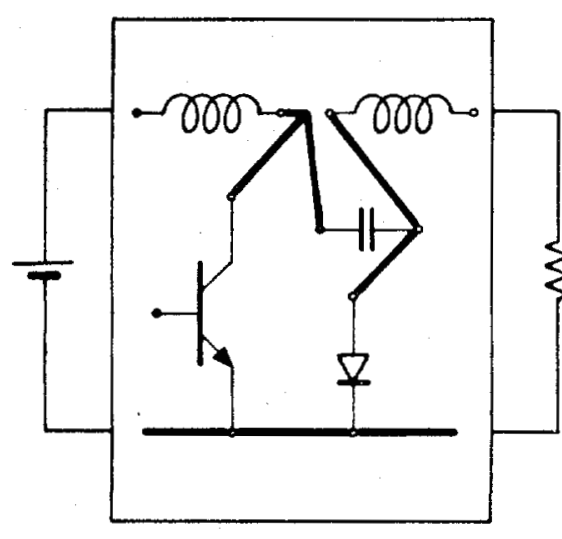

(a)

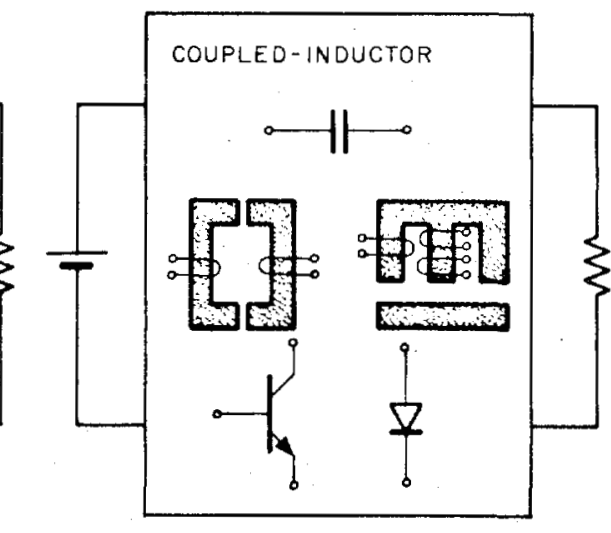

(b)

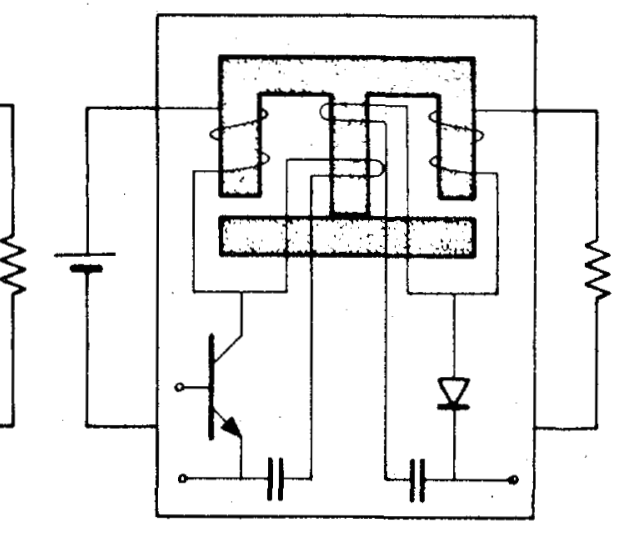

(c)

Fig. 1. Three key steps in switching converter developments. (a) Topology of electrical interconnections. (b) Topology of magnetic interconnections. (c) Blending of electrical and magnetic topology into single converter structure.

the possibility of reducing the size, weight, and cost of converter magnetic content, which is usually a dominant part in all switching converter configurations, without compromising converter performance, and in fact in some instances considerably improving it. As illustrated in Fig. 1(b), this has led to equal attention given to the magnetic interconnection of inductors and transformers, resulting in single magnetic structures with multiple windings, which emulate the function of several inductors and/or transformers. The final outcome in Fig. 1(c) is a converter in which switching converter electrical topology blends nicely with its magnetics topology to result in a simpler, less costly, and better performance configuration.

In Section II the coupled-inductor and integrated magnetics technique is briefly reviewed. Several new alternative core configurations are introduced in Section III and their relative merits discussed. The analysis of these complex magnetic structures is introduced in Section IV, which leads to the complete magnetics design (core sizes, number of turns, wire size, etc.) for the given specifications at hand. In Section $V$ future directions for experimental and theoretical (analytical) work are outlined.

\section{Review of Integrated Magnetics Concept}

We define the integrated magnetics concept as the process in which the inductors and transformers are merged into a single magnetic circuit with multiple windings. It is obvious that this is not always possible, since certain prerequisities have to be fulfilled beforehand. For example, the single-output version of the dc isolated Cuk converter has the necessary prerequisite: equal and proportional voltage waveforms not only on the inductors but on the primary and secondary of the isolation transformer, as illustrated in Fig. 2. This comes about because of the volt-second balance in steady state on all magnetic components, leading to a rectangular voltage waveform switching between input $\boldsymbol{V}_{\boldsymbol{g}}$ and output $-V$ dc voltage. Furthermore, with the change of operating point (duty cycle $D$ etc.) the waveforms automatically adjust to maintain the volt-second balance and hence proportionality is preserved despite source, load, or operating point changes. Thus, as explained at length in $[10]$ or $[4]$, all the prerequisites are satisfied to integrate the isolation transformer together with the two inductors into
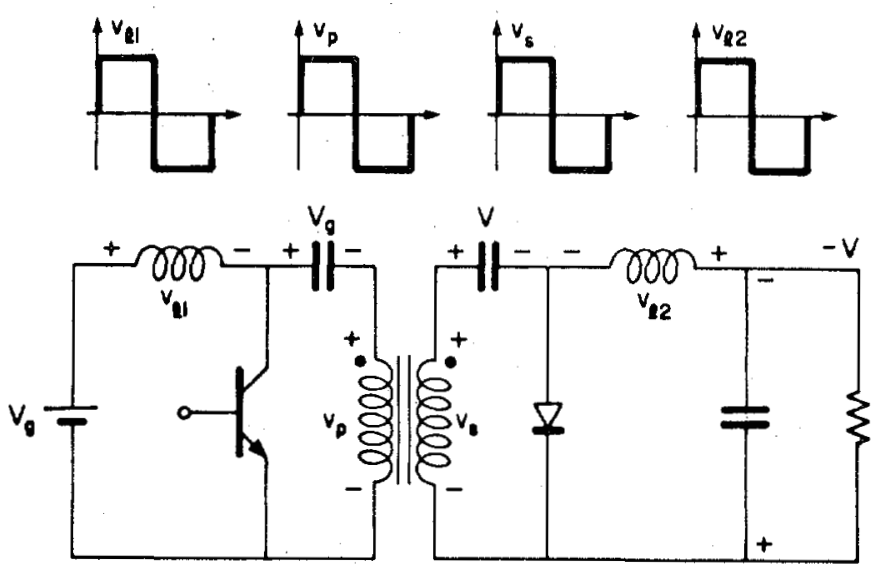

Fig. 2. DC isolated Cuk converter possesses unique feature of equal (proportional) voltage waveforms on all magnetic elements.

a single magnetic circuit with four windings, as illustrated in Fig. 3(a), by a simple extension of the line showing the magnetic core from the transformer into the two inductors. The particular magnetic core configuration is shown in Fig. 3(b). Note that in Fig. 3(a), the line showing the magnetic coupling through core is purposely shown not to connect input and output inductors directly but through the isolation transformer, as its core realization in Fig. 3(b) also suggests. Because the outer legs of the EI configuration containing inductors have air gaps, the flux generated by the ampere turns excitations on the input inductor, for example, closes through the inner leg of the EI core, which has a much smaller reluctance than the other outer leg containing the air gap. Hence, the input inductor is for all practical purposes coupled to the isolation transformer and not (or very little) directly to the output inductor. The same holds true for the output inductor, so that this coupling scheme is appropriately designated by the core notation adopted in Fig. 3(a).

From the conceptual viewpoint, this was the first time that inductors and transformers, previously exclusively used separately in switching structures, were now integrated into a single magnetic circuit with multiple windings.

Besides obvious simplification of the converter magnetic content (single core versus previous three cores), and corresponding savings in size and weight, one of the additional 

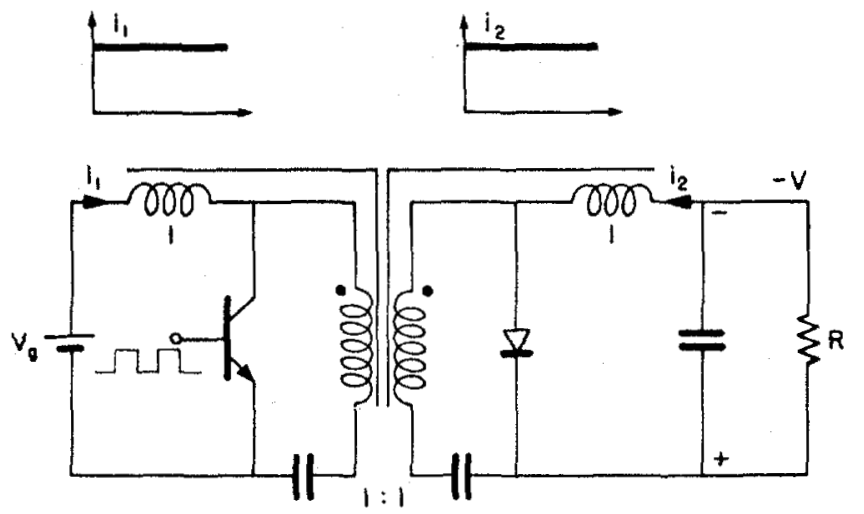

(a)
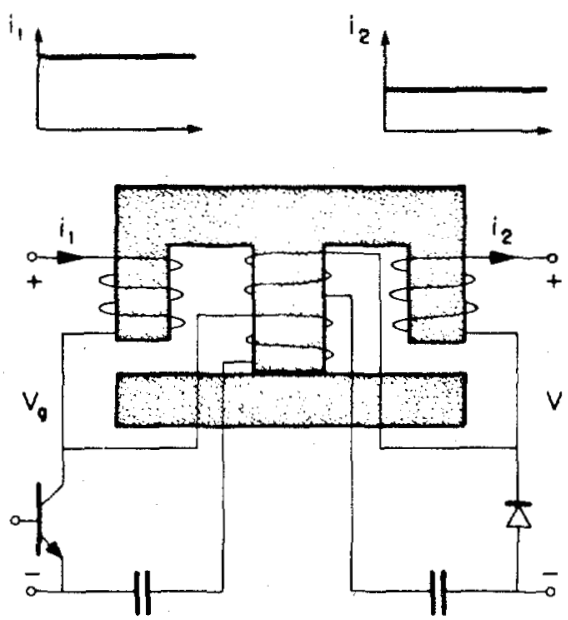

(b)

Fig. 3. (a) Zero-ripple Cuk converter. (b) Its single magnetic circuit realization.
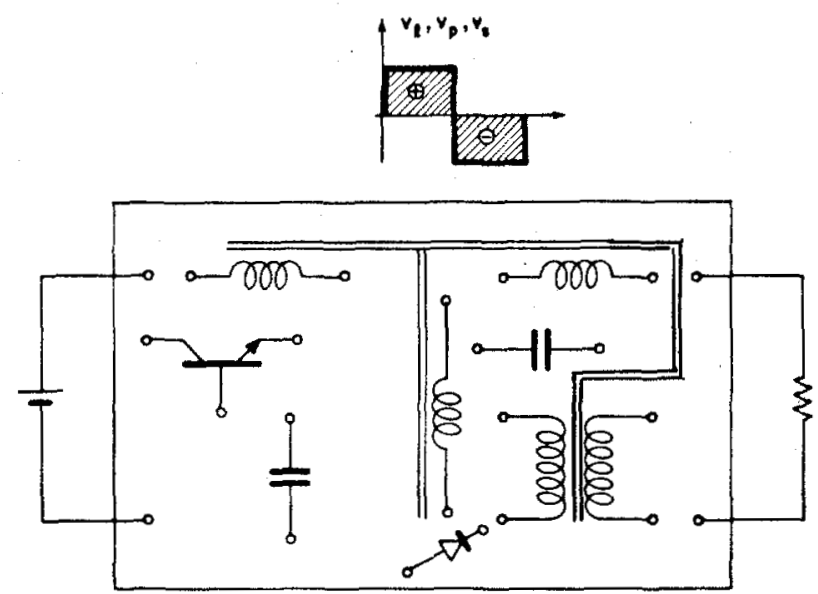

Fig. 4. Number of separate inductors and transformers can be merged into single magnetic circuit with multiple windings provided inductor and transformer voltage waveforms are proportional.

main advantages is virtual elimination of current ripple at both input and output sides, as illustrated in Fig. 3. Namely, by proper magnetics design the input and output current ripples are shifted into the isolation transformer windings, where they can be more readily tolerated (inside the black box). This concept may be now generalized as illustrated in Fig. 4 as follows.

In any switching structure, a number of separate inductors and transformers can be integrated into a single magnetic cir- cuit with multiple windings, provided the inductor and transformer voltage waveforms before coupling are in a fixed ratio of proportionality to one another, independent of the operating point.

Note that the line designating the magnetic core in Fig. 4 represents the general case in which all windings are coupled to one another. A multitude of alternative core structures exist when one starts to examine various air-gapped core structures and different coupling arrangements. Let us now explore some of them in more detail.

\section{New Alternative Magnetic Core Structures}

Although merging of the inductors and transformer into a single core configuration of Fig. 3 resulted in a simplified and zero-ripple configuration, it has also led to some undesirable drawbacks. For example, the isolation transformer in the converter of Fig. 2 (separate transformers and inductors) has the ideal zero dc bias feature (dc blocked by coupling capacitors on each side). In principle it can be built on square-loop ungapped core structures, hence resulting in a compact and efficient transformer with low leakages. However, when built in the EI configuration of Fig. 3(b), the isolation transformer "sees" the air gaps of both inductors. While this is helpful during a fast transient, preventing core saturation of the isolation transformer because of instantaneous volt-second imbalance during the charging of coupling capacitors, it does not serve any real purpose in the steady state.

\section{A. Three-Bobbins Single-Core Implementation}

An alternative EI core implementation which eliminates this drawback and maintains good isolation transformer features is shown in Fig. 5. This time, only the inner leg is gapped and carries the coupled inductors only, while the outer legs are ungapped and carry the isolation transformer windings. Thus as seen in Fig. 5(a), the transformer windings are virtually not coupled to the inductor windings. Note, however, that for better coupling each outer leg would be made to carry both primary and secondary windings of isolation transformer.

Furthermore, the presence of the inner gapped leg would prevent saturation of the transformer during fast transients, while still providing basically the ungapped configuration in the normal steady-state operation. The only drawback appears to be in fact that with the configuration of Fig. 5, the ripple current can be eliminated at only one end.

The low-leakage split primary and split secondary of the isolation transformer shown in Fig. 6 is commonly practiced with a UI core implementation of the isolation transformer only. The additional inner leg of the EI configuration then serves to realize the coupled-inductor configuration and provides the filtering at both input and output ends.

It is now apparent that either in the magnetics implementation of Fig. 5(b) or Fig. 6 the magnetic core provides the flux path for both the transformer flux $\phi_{t}$ and the coupled-inductors flux $\phi_{i}$, except for the inner leg which has mainly the coupledinductor flux $\phi_{i}$ and a negligible portion of the transformer flux $\phi_{t}$. In order to avoid core saturation, the total flux swing must be less than the saturation value $\phi_{s}$ for a given core crosssection and material type. Thus, for the outer legs, the follow- 


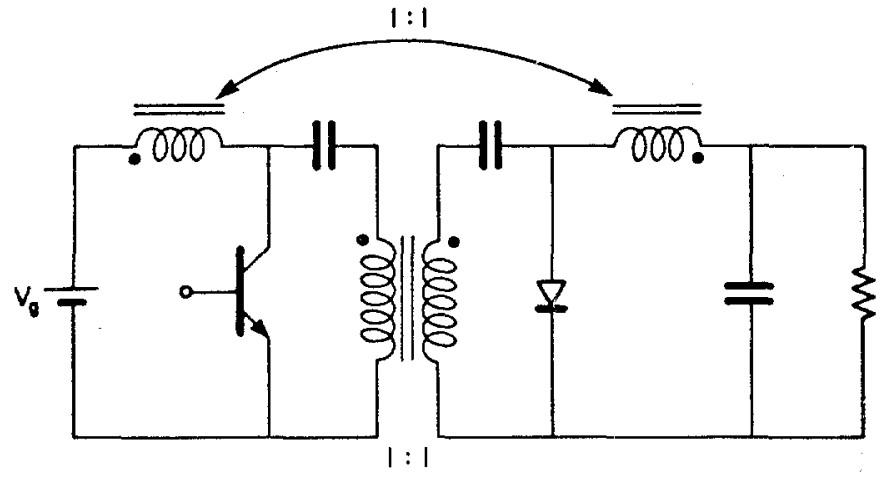

(a)

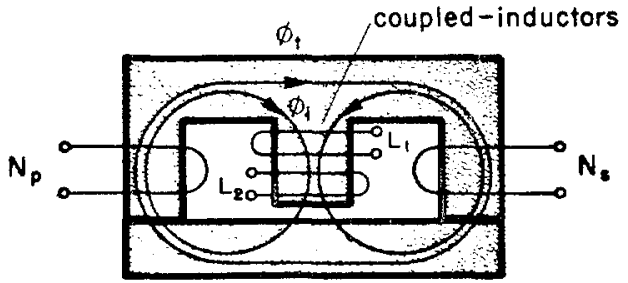

(b)

Fig. 5. (a) Coupled-inductor dc isolated Ćuk converter. (b) Its single EI core implementation using three bobbins.

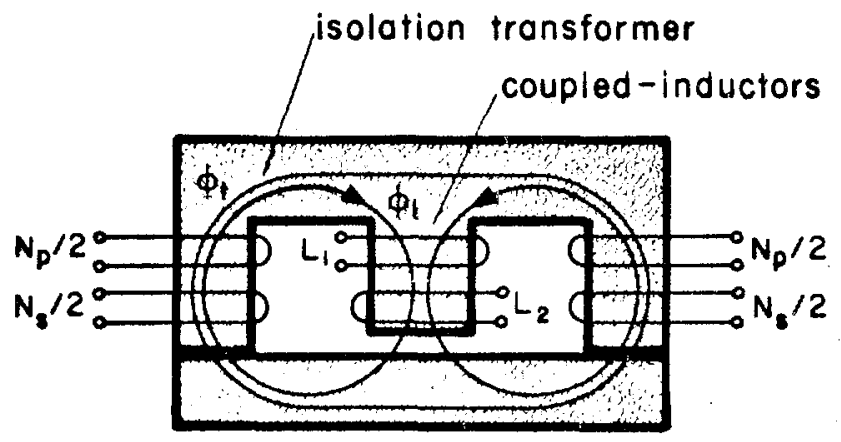

Fig. 6. Low leakage configuration of magnetic structure in Fig. 5.

ing limitation must be met regardless of the relative values of $\phi_{i}$ and $\phi_{t}$

$$
\phi_{i}+\phi_{t} \leqslant \phi_{s}
$$

since the inductor and transformer fluxes are additive.

The magnetic circuit design and optimization of this complex magnetic structure can now be reduced to the solution of two problems:

1) How do the inductors and transformer share the total flux swing $\phi_{s}$ available for the core of given cross-section? Alternatively, what are the portions of the total flux swing $\phi_{s}$ assigned to the inductor and transformer respectively?

2) How do the inductor and transformer windings share the available window area?

In particular, we are also interested to find out if there is an optimum choice of flux swing allocation, which will lead to the smallest core size and yet still achieve the required inductor and transformer conversion functions.

Let us now see how the relative flux allocations would affect the geometric configuration of the chosen EI core, that is, the relative cross-section area of the inner leg versus the outer legs. If the transformer flux $\phi_{t}$ is small and negligible compared to the inductor flux, the inner leg cross-section should be twice the area of the outer leg, to achieve best core utilization throughout the geometry of the core. This is so because the inductor flux divides into two equal parts.

Hence, the standard off-the-shelf EI core geometry could be used. However, if the inductor and transformer share the flux swing equally, then ideally all three legs should be of equal cross-sectioned area. Hence, the standard three-phase EI core geometries would be suitable. Any flux allocation in between these two limits would naturally have the inner cross-section appropriately adjusted within the limits of the single and double cross-section of the outer legs.

However this rather impractical solution can be completely avoided. Namely taking into account the converter circuit configuration of Fig. 5(a) and the possibility for saturation of the isolation transformer during fast transients, suggests the standard single-phase EI configuration. This is needed to protect against saturation of the inner leg when the transformer flux closes through it.

\section{B. Core Implementation with Orthogonal Fluxes}

Orthogonal fluxes had been investigated in the past for a variety of applications [11], [12]. Here we propose one such specific application for switching power conversion field. In the core configuration of Fig. 5, the two fluxes $\phi_{i}$ and $\phi_{t}$ are added in the same plane. However there is no reason why they could not be in different planes. In particular, when the two fluxes are in two perpendicular planes, ideally there is no coupling between them just as the circuit of Fig. 5(a) indicates, if the fluxes are exercised along linear portion of their $B-H$ characteristics. Yet there is a conceptually very interesting core realization using a single magnetic core as shown in Fig. 7, which actually leads to significantly better utilization of the core material.

In the case of the ferrite pot core, illustrated in Fig. 7(a), the two windings of the coupled-inductor are wound as usual on the bobbin and placed inside the gapped pot core. The isolation transformer windings are then added treating the pot core as a toroid and using the middle hole for the winding: the resultant flux density vector $B_{r}$ is the vectorial sum of the two perpendicular components $B_{i}$ and $B_{t}$, as illustrated in Fig. 7(a). As illustrated in Fig. 8 for the case of equal transformer and inductor flux densities $B_{t}$ and $B_{i}$, a full 70 percent of the saturation flux density $B_{s}$ (or $\sqrt{2} / 2 B_{s}=0.707 B_{s}$ to be more exact) is available for both transformer and inductor design, compared to only 50 percent in the case of their addition in a single plane as in previous case. Hence a better utilization of the core material is achieved. This would then translate into a lower loss, and more cost-effective design.

The implementation with the readily available pot core with a center hole, however, may not be very practical and suitable because of the rather odd area available for the transformer winding. Nevertheless, a specially constructed toroid having two halves shown in Fig. 7(b) could be a suitable solution. The isolation transformer windings then could be placed inside 

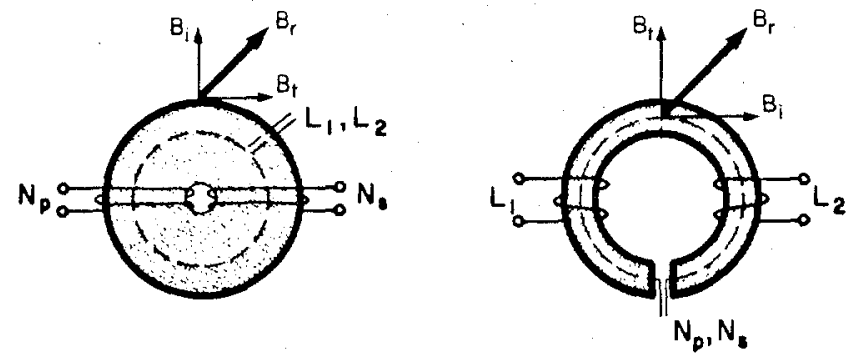

(a)
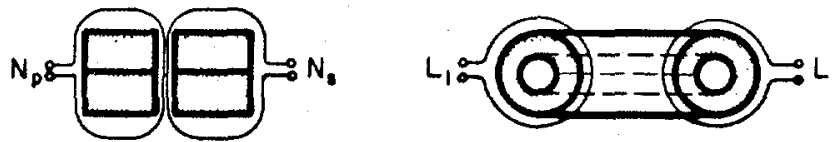

(b)

Fig. 7. Transformer and coupled-inductor fluxes in perpendicular arrangement lead to a better core utilization. (a) Pot core implementation. (b) Toroidal implementation.

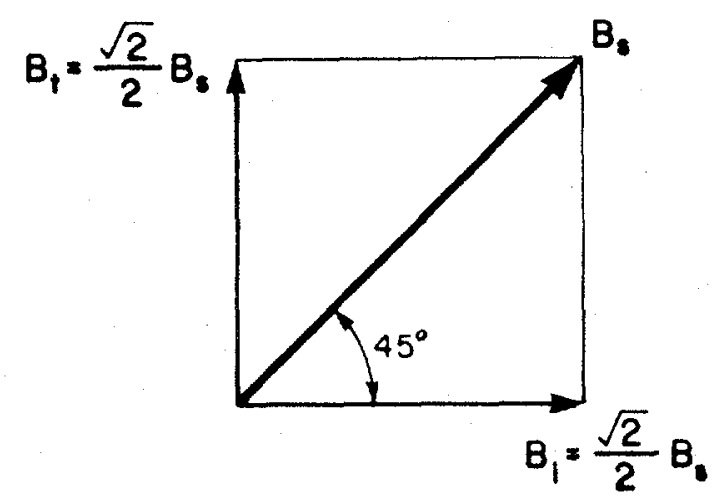

Fig. 8. Full 70 percent of total flux swing can be used by both transformer and coupled-inductors simultaneously.

the toroid, while the coupled-inductor windings could be wrapped around it in the usual fashion. For easier recognition, the magnetic cores are shown shaded in Fig. 7. The price paid for better utilization of the core material in the configuration of Fig. 7 is in greater complexity of the structure and relative inflexibility in making any changes in design after it is wound, compared to that of Fig. 6. Also the question of how close to an ideal isolation transformer with low leakages can be obtained with a configuration of Fig. 7 remains to be answered through experimental verification and analytical prediction. However irrespective of its final practical utility and usefulness, this example clearly demonstrates, at least conceptually, how the actual core geometry configuration and concept of sharing same magnetic flux paths among various magnetic components (inductors and transformers) can actually lead to better core utilization and more efficient design.

The experimental investigation of the orthogonal fluxes was very instrumental in discovering another very useful application for switching converters. During the experiment, it was found that the orthogonal fluxes do interact, due to the nonlinear nature of the core material. Therefore, an additional perpendicular winding can be used as a sense winding to detect the impending saturation of the transformer core in switching converters [13], and prevent their catastrophic

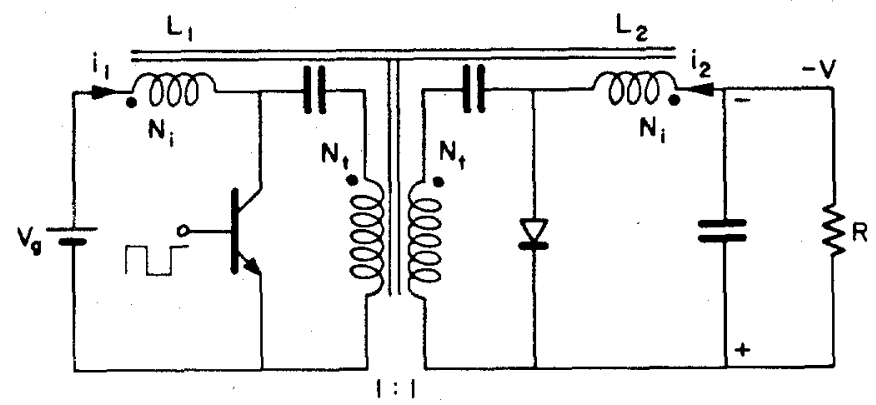

(a)

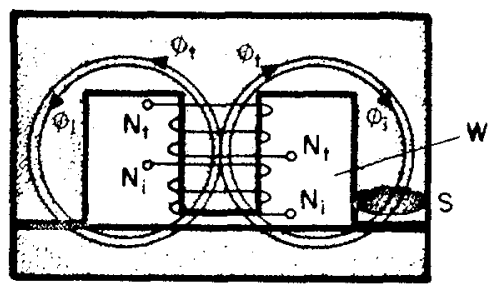

(b)

Fig. 9. (a) Single magnetic circuit will all four windings coupled on common core. (b) Single bobbin gapped EI core implementation.

failures. After this slight digression from our main stream, let us now look at some alternative integrated magnetics implementations.

\section{Single-Bobbin Single-Core Implementation}

While the implementation of Fig. 5 has many desirable features, it also has some practical disadvantages. For example, to implement the core configuration of Fig. 5, one needs three bobbins, all of which are nonstandard items since that type of core is exclusively used with the single center-leg bobbin. Thus, probably the simplest alternative, which does not require any specially gapped core (such as that in Fig. 3(b), nor any special bobbin arrangement, but uses standard off-theshelf components, is the one shown in Fig. 9(b).

Two isolation transformer windings with $N_{t}$ turns each, and two inductor windings with $N_{i}$ turns each are all put on a single bobbin and mounted on the center-leg of the EI core (Fig. 9(b)). For the implementation with a ferrite or squareloop core material the center-leg of the core is gapped to provide dc current capability. Although as before there are two fluxes, $\phi_{t}$ due to the transformer operation and $\phi_{i}$ due to inductor operation, each of the center-leg fluxes branches into half fluxes which travel to the outer legs. Thus regardless of the relative proportion of the two fluxes $\phi_{i}$ and $\phi_{t}$, the core cross-sections are optimally utilized with the center-leg having the standard double cross-section.

Note that, unlike in the previous case, the inductor windings are strongly coupled with the transformer windings as well as among themselves, as reflected on the circuit schematic of Fig. 9(a), showing core coupling (double lines) among all four windings. Note that the transformer and inductor windings are coupled in such a way that their ampere-turns add. The currents entering the dot signs (only one winding each is shown in Fig. 10 for simplicity) results in adding the two fluxes. Increasing ac flux in the inductor corresponds to transformer flux increasing in the same direction. Hence the same 


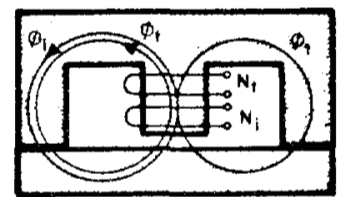

(a)

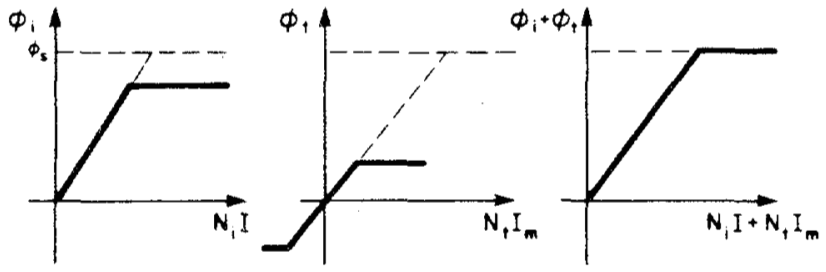

(b)

Fig. 10. Flux superposition in single-bobbin EI core configuration.

limitation as before (1) must be satisfied. This flux superposition is illustrated on Fig. 10(b), in which a core with same linear core material (and effective $\mu_{e}$ ) is used.

Finally, there is an additional limitation imposed by the coupling method itself. Note that as shown in Fig. 2, owing to the basic $1: 1$ relationship among transformer and inductor voltage waveforms before the couplings, the turns ratios are ideally limited to $1: 1: 1: 1$. Consequently one would require ideally equal numbers of turns on the inductors and the transformer, hence $N_{t}=N_{i}$. Because of the coupling among all four windings, the problem of determining how the switching current ripple divides among various windings is a formidable one, since it involves the modeling of leakages in a four-winding transformer. Nevertheless, it is possible that zero current ripple on both ends, or even only on one side, may not be achievable. Let us now try to derive some analy tical results for the two configurations proposed in Section III-B and Section III-C.

\section{Analysis of Complex Magnetic Structures}

For simplicity in presentation we will assume that the converter in Fig. 5 or Fig. 9 operates at $D=0.5$ duty cycle, resulting in current level $I$ in all four windings. Let us now briefly review the design equations.

\section{A. Transformer Design via Area Product $A_{p}$ Approach}

Here the same current density $J$ is adopted for both primary $N_{1}$ turns and secondary $N_{2}$ turns of the isolation transformer shown in Fig. 11. For a rectangular-wave periodic excitation, the primary voltage $V_{1}$ and secondary voltage $V_{2}$ which can be supported on a given core are obtained from Faraday's law as

$$
N_{1}=\frac{V_{1}}{4 B_{m} S f_{s}} ; \quad N_{2}=\frac{V_{2}}{4 B_{m} S f_{s}}
$$

where $B_{m}$ is the maximum allowable flux density and $f_{s}$ is the switching frequency.

Winding area $W$ is fully utilized when

$$
k W=N_{1} A w_{1}+N_{2} A w_{2}=N_{1} \frac{I_{1}}{J}+N_{2} \frac{I_{2}}{J}
$$

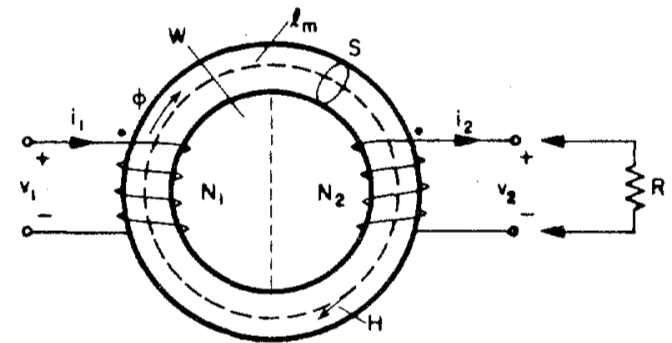

Fig. 11. Two winding isolation transformer shown schematically. In practice windings $N_{1}$ and $N_{2}$ are distributed around core to minimize leakage inductance.

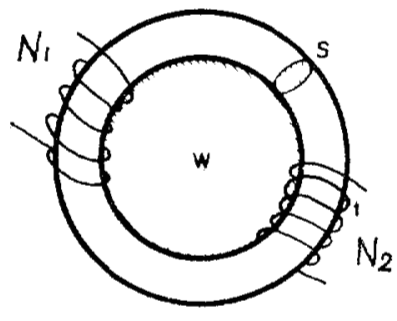

(a)

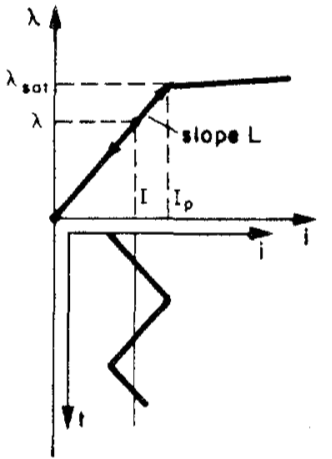

(b)
Fig. 12. (a) Definition of core geometry. (b) Design of coupled-inductors carrying dc current.

Substitution of $N_{1}$ and $N_{2}$ from (2) yields after rearrangement

$$
A_{p}=W S=\frac{V_{1} I_{1}+V_{2} J_{2}}{4 B_{m} k J f_{S}} .
$$

Usually a converter is designed for very high efficiency (on the order of 1-2 percent power loss). Thus $V_{1} I_{1} \approx V_{2} I_{2}$, and (4) becomes

$$
A_{p}=\frac{P}{2 B_{m} k J f_{s}}
$$

where $P$ is the power handling capability of the transformer.

\section{B. Coupled-Inductor Design via Area Product $A_{p}$ Approach}

The magnetic potentials of the two windings add in the coupled-inductor (Fig. 12) such that

$$
\left(N_{1} I_{1}+N_{2} I_{2}\right)=R \phi
$$

where $R=l_{m} / \mu S$ is the reluctance of the magnetic flux path and can be expressed as

$$
R=\frac{N_{1}^{2}}{L_{1}}
$$

where $L_{1}$ is self-inductance of the input winding. With this (6) becomes

$$
S=\frac{\left(N_{1} I_{1}+N_{2} I_{2}\right) L_{1}}{N_{1}^{2} B_{m}} .
$$

Full utilization of the window area results in

$$
W=N_{1} \frac{I_{1}}{k J}+N_{2} \frac{I_{2}}{k J} .
$$


Multiplying left and right sides of (8) and (9) we get

$$
A_{p}=W S=\frac{L}{2 B_{m} k J}\left(I_{1}+\frac{N_{2}}{N_{1}} I_{2}\right)^{2}
$$

where $L=2 L_{1}$ is the inductance which corresponds to the input current ripple before the coupling.

In the special case $I_{2}=0$ (single inductor)

$$
A_{p}=\frac{L I^{2}}{B_{m} k J} \text {. }
$$

In our case, however, $N_{1}=N_{2}$ and $I_{1}=I_{2}=I$ so

$$
A_{p}=\frac{2 L I^{2}}{B_{m} k J}
$$

or, the area product is only doubled from (11). To ensure that the core is not saturated:

$$
N_{1} I_{1}+N_{2} I_{2} \leqslant R B_{m} S
$$

In our special case of coupled-inductors $\left(D=0.5, N_{1}=N_{2}=N\right.$, $\left.I_{1}=I_{2}=I\right)$ we get

$$
N>\frac{L I_{p}}{B_{m} A_{c}}
$$

where $I_{p}=I+\Delta i / 2$ is the peak current.

\section{Transformer and Coupled-Inductor Designs Combined}

Three-Bobbin Single-Core Design: Let us now first analyze the three-bobbin alternative of Section III-A and Fig. 5 and Fig. 6. We assume that the transformer has $N_{t}$ primary and $N_{t}$ secondary turns (1:1 turns ratio) and coupled-inductors also $1: 1$ turns ratio but in general $N_{i}$ turns each where $N_{i} \neq N_{t}$. Finally, the transformer flux $\phi_{t}$ and inductor flux $\phi_{i}$ are assumed to be a fraction of the saturation flux $\phi_{s}$, thus

$$
\begin{aligned}
\phi_{t} & =\alpha \phi_{s} \\
\phi_{i} & =(1-\alpha) \phi_{s}
\end{aligned}
$$

where $\alpha$ is a free parameter. Through equal cross-sectional areas $S,(15)$ reduces to the relation for flux densities

$$
\begin{aligned}
B_{t} & =\alpha B_{m} \\
B_{i} & =(1-\alpha) B_{m} .
\end{aligned}
$$

To keep the transformer with $N_{t}$ turns excited just to $B_{t} \leqslant B_{m}$ flux density level, (2) becomes

$$
N_{t}=\frac{V_{g}}{4 B_{t} S f_{s}}=\frac{V_{g}}{4 \alpha B_{m} S f_{s}} .
$$

To keep the coupled-inductor flux excitation just to $B_{i} \leqslant B_{m}$ flux density level with $N_{i}$ turns, (14) becomes

$$
N_{i}=\frac{L I_{p}}{B_{i} S}=\frac{L I_{p}}{(1-\alpha) B_{m} S} .
$$

Since $B_{i}+B_{t}=B_{m}$, using $N_{t}$ and $N_{i}$ turns of (17) and (18), the full flux capability of the core is utilized.

Note that for now $\alpha$ remains a free parameter, that is how much flux swing is assigned to the transformer and how much to the coupled-inductor is yet to be determined. However, its range of change is apparently $\alpha \in(0,1)$. Boundaries $\alpha=0$, or $\alpha=1$ are not included (that directly excludes either transformer or coupled-inductor). The choice of $\alpha$ then directly affects the optimum number of turns $N_{t}$ and $N_{i}$. However we still have to fit both transformer $N_{t}$ and inductor $N_{i}$ turns into a single winding area $S$. Thus the additional constraint becomes

$$
k S=\dot{W}_{i}+W_{t}=2 N_{i} \frac{I}{J}+2 N_{t} \frac{I}{J}
$$

where $k$ is as before, the window utilization factor (fill factor) typically in the range of 0.4 (ferrite pot cores) to 0.6 (toroidal cores). Note also that the same current density is assumed for both transformer and inductor windings.

The substitution of (17) and (18) into (19) leads after rearrangement to

$$
A_{p}=\dot{W} S=\frac{2 L I I_{p}}{(1-\alpha) B_{m} k J}+\frac{V_{g} I}{2 \alpha B_{m} k J f_{s}} .
$$

This can be put in the form:

$$
A_{p}=\frac{A_{p i}}{1-\alpha}+\frac{A_{p t}}{\alpha}
$$

where

$$
A_{p i}=\frac{2 L I I_{p}}{B_{m} k J} ; \quad A_{p t}=\frac{V_{g} I}{2 B_{m} k J f_{s}} .
$$

The area products $A_{p i}$ and $A_{p t}$ in (22) are minimum area product requirements if inductor and transformer were built separately. From (22) another interesting relation is obtained:

$$
\frac{A_{p t}}{A_{p i}}=\frac{V_{g}}{4 L f_{s} I_{p}}=\frac{\Delta i / 2}{I_{p}}=\frac{\Delta i / 2}{I+\Delta i / 2}
$$

since the absolute ripple is $\Delta i=V_{g} D T_{s} / L$ :

Thus, if the separate inductors are to provide small ripple current (relative to its average dc value) in the same converter with a separate isolation transformer, the coupled-inductor area product (hence its size) is substantially larger than that needed for the transformer.

Note that once the electrical requirements are specified (ripple current $\Delta i$; dc current level $I$, power $V_{g} I$, switching frequency $f_{s}$ ) as well as design parameters ( $B_{m}$ for given core material, and current density $J$ ), both $A_{p t}$ and $A_{p i}$ are uniquely determined from (22). However the overall area product $A_{p}$ for combined inductor and transformer is still undetermined, since it is a strong function of the parameter $\alpha$, as seen in Fig. 13, which is a plot of its functional dependence (21). Namely, for $\alpha \rightarrow 0$ and $\alpha \rightarrow 1, A_{p} \rightarrow \infty$. Since in that range it is a positive quantity and does not have discontinuities, it has a minimum.

By use of calculus on (21)

$$
\frac{d A_{p}(\alpha)}{d \alpha}=\frac{\alpha^{2} A_{p i}-(1-\alpha)^{2} A_{p t}}{(1-\alpha)^{2} \alpha^{2}}=0
$$

or

$$
\frac{\alpha_{\mathrm{opt}}}{1-\alpha_{\mathrm{opt}}}=\frac{A_{p t}}{A_{p i}} .
$$




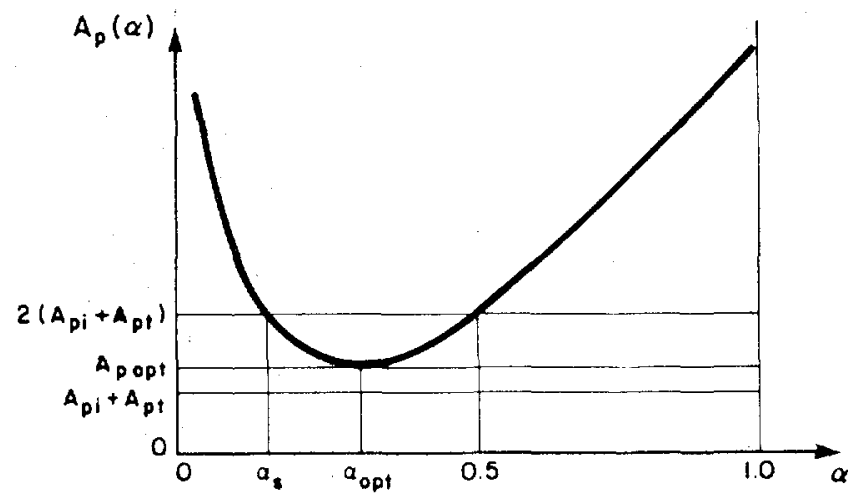

Fig. 13. Area product dependence on $\alpha \triangleq B_{t} / B_{m}$.

Thus the minimum size of the inductor, transformer magnetic circuit is obtained when the ratio of their flux utilizations is

$$
\frac{B_{t}}{B_{i}} /_{\text {opt }}=\frac{A_{p t}}{A_{p i}} .
$$

The optimum value $A_{p \text { opt }}$ is obtained by use of (25) in (21) as

$$
A_{p o p t}=\left(\sqrt{A_{p i}}+\sqrt{A_{p t}}\right)^{2}=A_{p i}+A_{p t}+2 \sqrt{A_{p i} A_{p t}}
$$

and for

$$
\alpha_{\mathrm{opt}}=\frac{\sqrt{A_{p t}}}{\sqrt{A_{p t}}+\sqrt{A_{p i}}} .
$$

Note that this optimum value is somewhat larger than if we simply sum the area products of inductors and transformers when built separately, that is

$$
A_{\text {popt }}>A_{p} \text { sum }=A_{p t}+A_{p i} .
$$

However the larger area product is not a direct indication of worse volumetric design. For example, if we build two separate toroidal inductors with each having area product $A_{p}=W S$, building them on a common core with two toroids stacked (coupled-inductor) will result in doubled area product. However as shown in (10) or (4), the coupled-inductor case actually results in almost twice smaller size than the two separate inductors.

For the special case of $\alpha=0.5$,

$$
A_{p}(\alpha)=2\left(A_{p i}+A_{p t}\right)
$$

so the optimum area product can be bounded on both sides through the inequality

$$
2\left(A_{p i}+A_{p t}\right)>A_{p o p t}>A_{p i}+A_{p t} .
$$

Finally, the total combined area product $A_{p}$ can be expressed in another useful form as

$$
A_{p}=\frac{A_{p t}}{\alpha}\left(1+\frac{2 I_{p}}{\Delta i} \frac{\alpha}{1-\alpha}\right)
$$

where result (23) was used in (32).

Note that in all of the above analysis, the general case was considered such that inductor $N_{i}$ and transformer $N_{t}$ turns were different. As a consequence the parameter $\alpha$ was a free parameter and the above optimization analysis was carried out.
However, when coupled-inductors are also magnetically coupled to the isolation transformer, $\alpha$ is not a free parameter. This is so in our other single bobbin alternative of Section III-C for which $N_{t}=N_{i}$ because of the $1: 1$ turns ratio of transformer to inductor coupling.

\section{Analysis of Single-Bobbin Single-Core Configuration}

For the magnetic circuit of Fig. 13, an additional requirement is imposed owing to the $1: 1: 1: 1$ turns ratio condition as

$$
\frac{N_{t}}{N_{i}}=1
$$

However from (17) and (18)

$$
\frac{N_{t}}{N_{i}}=\frac{\Delta i}{2 I_{p}} \frac{1-\alpha}{\alpha}=1
$$

Thus the parameter $\alpha$ is no longer free but is directly dictated by the choice of the relative ripple current in the inductors, that is, $\alpha=\alpha_{s}$ and

$$
\frac{\alpha_{s}}{1-\alpha_{s}}=\frac{\Delta i}{2 I_{p}}
$$

The corresponding area product $A_{p}\left(\alpha_{s}\right)$ becomes

$$
A_{p}\left(\alpha_{s}\right)=\frac{A_{p t}}{\alpha_{s}}(1+1)=\frac{2 A_{p t}}{\alpha_{s}}
$$

which indicates that the area products and, because of the common cross-section $S$, the window areas allocated to transformer and inductors are equal (half usable window area). This is, of course, under the assumption of equal current density $J$ in transformer and inductors.

Using (23) and (35), (36) also can be expressed as

$$
A_{p}\left(\alpha_{s}\right)=2\left(A_{p t}+A_{p i}\right) ; \quad \alpha_{s}=\frac{A_{p t}}{A_{p i}+A_{p t}}
$$

which is the same as obtained for $\alpha=0.5$ as seen from (30) and shown in Fig. 13. Although this is clearly worse than the optimum value given by (27), the following example demonstrates that it is quite close to the minimum value of the previous design. For typical values

$$
\begin{aligned}
A_{p i} & =9 A_{p t} \\
A_{p o p t} & =16 A_{p t} \\
A_{p}\left(\alpha_{s}\right) & =20 A_{p t} .
\end{aligned}
$$

Hence, it is only 25 percent higher than the minimum possible value with the previous design.

Finally, a note about the magnetizing current $i_{m}$ of the isolation transformer in this design. Assuming a linear flux versus ampere-turns characteristic (a ferrite core with an air gap is very closely linear) we have

$$
\phi_{t}=\alpha \phi_{s} \quad N \frac{i_{m}}{2}=\alpha N I_{s} \quad i_{m}=2 \alpha I_{s}
$$


or

$$
i_{m}=\frac{2 \alpha}{1-\alpha} I=\frac{\Delta i I}{I_{p}}=\Delta i .
$$

Thus the magnetizing current and the ripple current in inductors are equal. Hence in order to reduce magnetizing current, a design parameter ripple current in inductors has to be chosen small enough (say 10-20 percent).

\section{CONCLuSion}

Several new and promising magnetic circuit configurations are proposed as alternative implementations of the integrated magnetics approach introduced previously. [4], [10]. These magnetic structures for the first time combine the inductors and transformers on a single magnetic core. The analytical treatment is somewhat more complex, but the benefits in more efficient use of core material, smaller, more efficient, and compact designs certainly far outweigh any analysis difficulties. Nevertheless a simplified analysis covering only firstorder effects is easily carried out, and equations are developed for the design of these complex magnetic structures. In one particular configuration with three bobbins even an optimization can be carried out to determine the most favorable ratio of flux sharing between the inductors and transformers, such that the resultant designs give the lowest overall size.

Experimental verification of these and some other alternatives is currently in process, as well as determination of how. some second-order effects (such as leakage inductances) affect overall converter performance.

\section{REFERENCES}

[1] S. Cuk, "Modelling, analysis and design of switching converters," Ph.D, dissertation, California Institute of Technology, Nov. 1976. Also, NASA Report CR-135174.

[2] L. Rensink, "Switching regulator configurations and circuit realizations," Ph.D. dissertation, California Institute of Technology, Dec. 1979.

[3] R. D. Middlebrook and S. Cuk, Advances in Switched-Mode Power Conversion, vol. I. Pasadena, CA: TESLAco, 1981. (Available from TESLAco, 490 S. Rosemead Blvd., Suite 6 , Pasadena, CA 91107.)

[4] S. Cuk and R. D. Middlebrook, Advances in Switched-Mode Power Conversion, vol. II. Pasadena, CA: TESLAco, 1981.

[5] — "Dc-to-de switching converter," U.S. Patent \#4,184,197, Jan. 1980. Foreign patents pending.

[6] S. Cuk, "Push-pull switching power amplifier," U.S. Patent $\# 4,186,437, \mathrm{~J}$ an: 1980 . Foreign patents pending.

[7] S. Cuk and $\dot{R}$. D. Middlebrook, "Dc-to-dc converter having reduced ripple without need for adjustments," U.S. Patent $\# 4,274,133$, June 1981.

[8] S. 'Cuk, "Dc-to-dc switching converter with zero input and output current ripple and integrated magnetics circuits," U.S. Patent \#4,257,087, Mar. 1981.

[9] - "Switching dc-to-dc converter with zero input or output current ripple," in Proc. IEEE Ind. Appl. Soc. Annu. Meeting, 1978, pp. 1131-1146.

[10] -, "A new zero-ripple switching dc-to-dc converter and integrated magnetics," in Proc. IEEE Power Electronics Specialists Conf., 1980 , pp. 12-32.

[11] R. A. Heartz and H. Buelteman, Jr., "The application of perpendicularly superimposed magnetic fields," AIEE Trans., Part I, vol. 74, pp. 655-660, Nov. 1955.

[12] F. C. Schwarz, "An unorthodox transformer for free running parallel inverters," IEEE Trans. Magn., vol. MAG-5, no. 4, pp. 908-912, 1969

[13] W. M. Polivka, A. Cocconi, and S. Cuk, "Detection of magnetic saturation in switching converters," in Proc. Fourth Annual International $P C T^{\prime} 82$ Conf., 1982, pp. 583-598. 